\title{
COMMENT
}

\section{A family of NICU graduates!}

\author{
Praveen Chandrasekharan ${ }^{1}$ \\ Pediatric Research (2018) 84:578-579; https://doi.org/10.1038/s41390-018-0157-y
}

My parents, an engineer and a physician, inspired me to pursue science and medicine while growing up in Chennai, India. Witnessing umpteen number of cases with birth asphyxia and consequent long-term neurological complications during my training left an everlasting impression on me. These cases influenced my decision to pursue a career in academic pediatrics and, subsequently, neonatology.

I have been fortunate to work with several exceptional mentors, beginning with Dr. Kusum Viswanathan, at Brookdale Hospital in New York, where I completed my pediatric residency. From clinical pediatrics to basic statistics, she instilled in me an understanding of the core concepts of research.

During my residency, I met my wife, Munmun, a constant source of inspiration, and a neonatologist. Together we worked with Dr. Roger Kim on quality improvement projects and collaborated in a multicenter non-invasive ventilation trial. ${ }^{1}$

The turning point in my career was meeting Dr. Satyan Lakshminrusimha, a pioneer in translational research and my role model. During my neonatology fellowship at the University at Buffalo (UB), under his guidance, I worked on both basic science and clinical research. ${ }^{2-21}$ Our lab, complete with a team of experienced personnel, is one of the handful centers in the US conducting perinatal translational research.

My greatest motivation was the birth of my son, Ishaan. He was born premature and has benefitted from every aspect of neonatal and pediatric research, which further inspires me to conduct research addressing prematurity and its problems.

Currently, my work focuses on studying the effects of gas exchange on pulmonary and systemic hemodynamics in both preterm and term models during and post-resuscitation. My recently published research, investigated the impact of oxygen used during preterm resuscitation, an area that continues to be controversial. ${ }^{7}$ This research earned me a young investigator award at the Eastern Society of Pediatric Research.

As a junior faculty at UB, I have evaluated the effect of placental transfusion during preterm resuscitation (supported by Buswell, UBMD \& Kaleida Health). ${ }^{11}$ Also, I am assessing ways to improve the efficiency of neonatal resuscitation, a project funded by AAP NRP. With a bioinformatics background, I am fortunate to be part of the neonatal research network alongside Dr. Anne Marie Reynolds. Moving forward, I intend to explore the combined impact of oxygen use and placental transfusion during preterm resuscitation.

I urge young researchers to find supportive mentors and colleagues. I would not be where I am today without them.
Photo: Praveen Chandrasekharan, Munmun Rawat and Ishaan Praveen

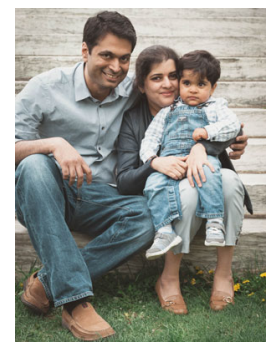

Photo: Praveen Chandrasekharan

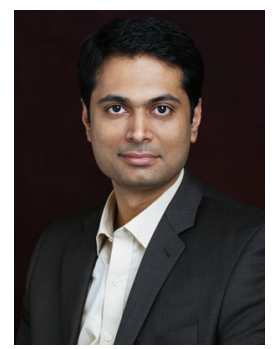

\section{REFERENCES}

1. Kirpalani, H. et al. A trial comparing noninvasive ventilation strategies in preterm infants. N. Engl. J. Med. 369, 611-620 (2013).

2. Chandrasekharan, P., Konduri, G., Basir, M., Klein, J. \& Lakshminrusimha, S. Risk stratification for congenital diaphragmatic hernia-is it all oxygenation but not ventilation? J. Perinatol. 38, 608-609 (2018).

3. Chandrasekharan, P. et al. Early use of inhaled nitric oxide in preterm infants: is there a rationale for selective approach? Am. J. Perinatol. 34, 428-440 (2017).

4. Chandrasekharan, P. \& Lakshminrusimha, S. The effectiveness of oral dextrose gel for the treatment of neonatal hypoglycaemia remains unclear. Evid. Based. Nurs. 20, 80-81 (2017).

5. Chandrasekharan, P. \& Lakshminrusimha, S. Single dose of prophylactic oral dextrose gel reduces neonatal hypoglycaemia. Evid. Based. Med. 22, 62 (2017).

6. Chandrasekharan, P., Nrusimha, A., Rawat, M., Lakshminrusimha, S. Using paralytic as part of premedication for elective intubation of premature neonates may result in transient impairment of ventilation. Am. J. Perinatol. (2018)

7. Chandrasekharan, P., et al. Effect of various inspired oxygen concentrations on pulmonary and systemic hemodynamics and oxygenation during resuscitation in a transitioning preterm model. Pediatr. Res. (2018) 
8. Chandrasekharan, P., Rawat, M. \& Lakshminrusimha, S. Complications of prematurity: an infographic. J. Perinatol. 37, 1185-1186 (2017).

9. Chandrasekharan, P., Rawat, M., Reynolds, A. M., Phillips, K. \& Lakshminrusimha, S. Apnea, bradycardia and desaturation spells in premature infants: impact of a protocol for the duration of 'spell-free' observation on interprovider variability and readmission rates. J. Perinatol. 38, 86-91 (2018).

10. Chandrasekharan, P. et al. Continuous capnography monitoring during resuscitation in a transitional large mammalian model of asphyxial cardiac arrest. Pediatr. Res. 81, 898-904 (2017).

11. Chandrasekharan, $P$., et al. Placental transfusion during resuscitation of a partially asphyxiated preterm model. Pediatr. Acad. Soc. Meet. 1725.8. (2018)

12. Chandrasekharan, P. K., Rawat, M., Madappa, R., Rothstein, D. H. \& Lakshminrusimha, S. Congenital diaphragmatic hernia - a review. Matern. Health Neonatol. Perinatol. 3, 6 (2017).

13. Chandrasekharan, P. K. et al. Continuous end-tidal carbon dioxide monitoring during resuscitation of asphyxiated term lambs. Neonatology. 109, 265-273 (2016).

14. Rawat, M., et al. Oral dextrose gel reduces the need for intravenous dextrose therapy in neonatal hypoglycemia. Biomed. Hub 1, pii: 448511 (2016).
15. Rawat, M. et al. Neonatal resuscitation adhering to oxygen saturation guidelines in asphyxiated lambs with meconium aspiration. Pediatr. Res. 79 583-588 (2016).

16. Rawat, M. et al. Oxygen saturation index and severity of hypoxic respiratory failure. Neonatology. 107, 161-166 (2015).

17. Rawat, M., Nangia, S., Chandrasekharan, P. \& Lakshminrusimha, S. Approach to infants born through meconium stained amniotic fluid: evolution based on evidence? Am. J. Perinatol. 35, 815-822 (2018).

18. Singh, A. P., Balluz, R. \& Chandrasekharan, P. Poor oral intake in a late preterm twin - usual symptom with an unusual diagnosis. Heart Lung 47, 162-165 (2018).

19. Vali, P., et al. Evaluation of timing and route of epinephrine in a neonatal model of asphyxial arrest. J. Am. Heart Assoc. 6, pii: e004402 (2017).

20. Vali, P. et al. Continuous chest compressions during sustained inflations in a perinatal asphyxial cardiac arrest lamb model. Pediatr. Crit. Care. Med. 18, e370-e377 (2017).

21. Vali, P. et al. Hemodynamics and gas exchange during chest compressions in neonatal resuscitation. PLOS ONE 12, e0176478 (2017). 